ORNL/TM-2000/93

\title{
COMPUTATION OF NORMAL CONDUCTING AND SUPERCONDUCTING LINEAR ACCELERATOR (LINAC) AVAILABILITIES
}

\author{
M. Jonathan Haire \\ Oak Ridge National Laboratory \\ Oak Ridge, Tennessee 37831-6180 \\ Tel: (865) 574-7141 \\ Fax: (865) 574-9512 \\ E-mail: hairemj@ornl.gov \\ Jack C. Schryver \\ Oak Ridge National Laboratory \\ Oak Ridge, Tennessee 37831-6364 \\ Tel: (865) 574-4710 \\ Fax: (865) 241-0381 \\ E-mail: schryverjc@ornl.gov \\ December 1999 \\ Prepared by \\ Oak Ridge National Laboratory \\ Oak Ridge, Tennessee 37831 \\ under contract DE-AC05-00OR22725 \\ Prepared for the \\ U.S. Department of Energy \\ Office of Science \\ UT-BATTELLE, LLC \\ managing the \\ Spallation Neutron Source Activities at the \\ Argonne National Laboratory \\ Brookhaven National Laboratory \\ Lawrence Berkeley National Laboratory \\ Los Alamos National Laboratory \\ Oak Ridge National Laboratory \\ under contract DE-AC-05-96OR22464 \\ for the \\ U.S. DEPARTMENT OF ENERGY
}




\section{CONTENTS}

LIST OF FIGURES $\ldots \ldots \ldots \ldots \ldots \ldots \ldots \ldots \ldots \ldots \ldots \ldots \ldots \ldots \ldots \ldots \ldots \ldots \ldots \ldots$

LIST OF TABLES $\ldots \ldots \ldots \ldots \ldots \ldots \ldots \ldots \ldots \ldots \ldots \ldots \ldots \ldots \ldots \ldots \ldots \ldots \ldots \ldots$

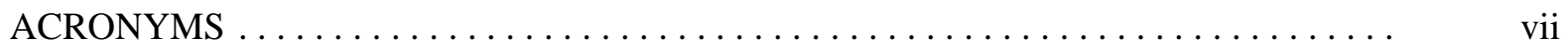

EXECUTIVE SUMMARY $\ldots \ldots \ldots \ldots \ldots \ldots \ldots \ldots \ldots \ldots \ldots \ldots \ldots \ldots \ldots \ldots \ldots \ldots$

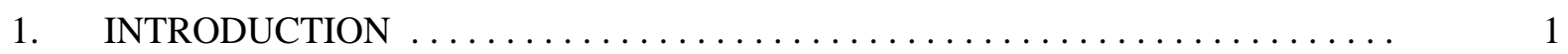

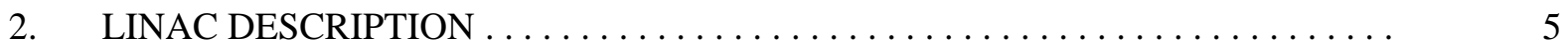

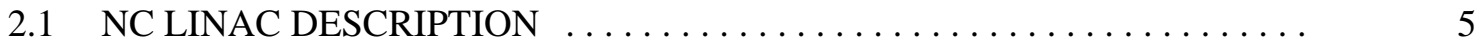

2.2 SC LINAC DESCRIPTION $\ldots \ldots \ldots \ldots \ldots \ldots \ldots \ldots \ldots \ldots \ldots \ldots \ldots \ldots \ldots \ldots \ldots$

3. TABULAR FAULT-TREE CALCULATIONS $\ldots \ldots \ldots \ldots \ldots \ldots \ldots \ldots \ldots$

$3.1 \quad$ FAULT-TREE DEVELOPMENT $\ldots \ldots \ldots \ldots \ldots \ldots \ldots \ldots \ldots \ldots \ldots \ldots \ldots$

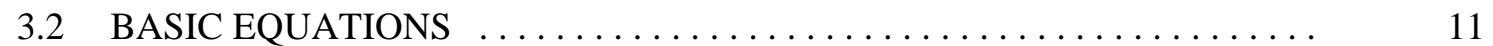

3.3 TABULAR FAULT-TREE CALCULATIONS $\ldots \ldots \ldots \ldots \ldots \ldots \ldots \ldots$

4. COMPUTER SIMULATION OF LINAC AVAILABILITY $\ldots \ldots \ldots \ldots \ldots \ldots \ldots$

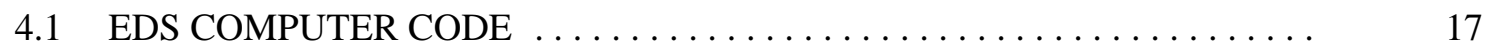

4.2 THE EFFECTS OF CAVITY FAILURE LOCATION AND HVPS
CONFIGURATION $\ldots \ldots \ldots \ldots \ldots \ldots \ldots \ldots \ldots \ldots \ldots \ldots \ldots \ldots \ldots \ldots$

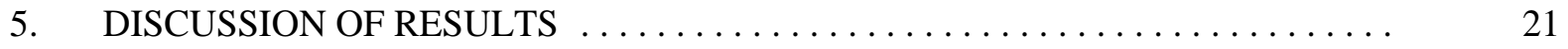

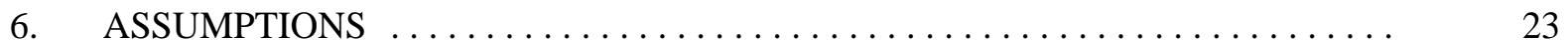

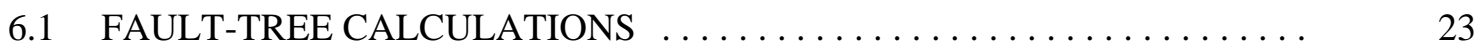

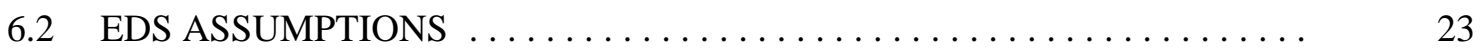

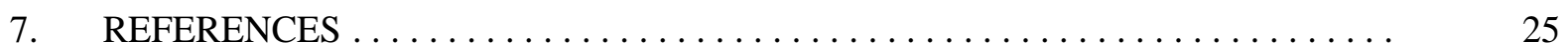




\section{LIST OF FIGURES}

Fig. $1 \quad$ Schematic of sections of SC LINAC $\ldots \ldots \ldots \ldots \ldots \ldots \ldots \ldots \ldots \ldots \ldots \ldots \ldots \ldots$

Fig. 2 Schematic of assumed NC linac module. (Each module contains 1 klystron servicing $8 \mathrm{rf}$ cavities with 10 cells per cavity, and associated equipment.) .....

Fig. 3 Schematic of assumed SC LINAC module. (Each module contains $3 \mathrm{rf}$ cavities, each driven by a klystron.) $\ldots \ldots \ldots \ldots \ldots \ldots \ldots \ldots \ldots \ldots \ldots \ldots \ldots \ldots \ldots \ldots \ldots \ldots$

Fig. 4. Fault tree for LINAC arrangement, where every klystron drives an SC cavity . . $\quad 10$

Fig. 5. The effect of klystron HVPS configuration on SC availability $\ldots \ldots \ldots \ldots \ldots$

\section{LIST OF TABLES}

Table 1 Components and data for NC LINAC section (- 200 to $1,000 \mathrm{MeV}) \ldots \ldots \ldots .2$

Table 2 Components and data for SC modules in LINAC section (- 200 to 1,000 MW) . 3

Table 3 Components and data for SC LINAC with six cavities per klystron $\ldots \ldots \ldots . \quad 15$ 


\section{ACRONYMS}

$\begin{array}{ll}\text { APT } & \text { Accelerator Production of Tritium } \\ \text { EDS } & \text { event driven simulation } \\ \text { HVPS } & \text { high voltage power supply } \\ \text { LINAC } & \text { linear accelerator } \\ \text { LLRF } & \text { low-level radio frequency } \\ \text { MTBF } & \text { mean time between failure } \\ \text { MTTR } & \text { mean time to repair } \\ \text { NC } & \text { normal conducting } \\ \text { rf } & \text { radio frequency } \\ \text { SC } & \text { superconducting } \\ \text { SNS } & \text { Spallation Neutron Source }\end{array}$




\section{EXECUTIVE SUMMARY}

A brief study was conducted to roughly estimate the availability of a superconducting (SC) linear accelerator (LINAC) as compared to a normal conducting (NC) one. Potentially, SC radio frequency cavities have substantial reserve capability, which allows them to compensate for failed cavities, thus increasing the availability of the overall LINAC. In the initial SC design, there is a klystron and associated equipment (e.g., power supply) for every cavity of an SC LINAC. On the other hand, a single klystron may service eight cavities in the NC LINAC. This study modeled that portion of the Spallation Neutron Source LINAC (between 200 and 1,000 MeV) that is initially proposed for conversion from NC to SC technology. Equipment common to both designs was not evaluated. Tabular fault-tree calculations and computer-event-driven simulation (EDS) computer computations were performed.

The estimated gain in availability when using the SC option ranges from 3 to $13 \%$ under certain equipment and conditions and spatial separation requirements. The availability of an NC LINAC is estimated to be $83 \%$. Tabular fault-tree calculations and computer EDS modeling gave the same $83 \%$ answer to within one-tenth of a percent for the NC case. Tabular fault-tree calculations of the availability of the SC LINAC (where a klystron and associated equipment drive a single cavity) give 97\%, whereas EDS computer calculations give $96 \%$, a disagreement of only $1 \%$. This result may be somewhat fortuitous because of limitations of tabular fault-tree calculations. For example, tabular fault-tree calculations can not handle spatial effects (separation distance between failures), equipment network configurations, and some failure combinations.

EDS computer modeling of various equipment configurations were examined. When there is a klystron and associated equipment for every cavity and adjacent cavity, failure can be tolerated and the SC availability was estimated to be $96 \%$. SC availability decreased as increased separation distance between failures is required for operation. Another configuration is a power supply servicing two or more klystrons and associated equipment. It makes a tremendous difference in availability if a power supply services adjacent klystrons, every other klystron, every third klystron, etc. The SC availability plummets to $-82 \%$ if the required separation distance between failures is 1 or 2 and the high voltage power supply (HVPS) services adjacent klystrons. However, if the HVPS drives alternate klystrons, a required separation of one cavity between failures can be tolerated.

One proposed SC LINAC design configuration has one power supply driving six cavities. In this configuration, any component failure will likely cause LINAC shutdown. The estimated availability for these conditions is $86.3 \%$. This gain over $83 \% \mathrm{NC}$ availability results from the higher availability of the $\mathrm{SC}$ tuner system. 
Some conclusions of this study are:

- An SC LINAC availability can be substantially (-13\%) higher than an NC LINAC if the capability of the SC LINAC to tolerate cavity failure is harnessed by configurations that allow operation with failure. About $6 \%$ of this increase in availability is caused by lower SC tuner system failure rates and repair times.

- Quadrupole magnets and power supply are primary contributor to SC LINAC unavailability.

- Power supply to klystrons and the tuner resonance control system are primary contributor to NC LINAC unavailability.

In summary, an SC LINAC availability is not inherently higher than an NC LINAC. SC availabilities can be made substantially higher than an NC LINAC availability if the design capitalizes on advantages offered by certain equipment configurations. Even though input failure rate and repair data to these calculations may be uncertain, this analysis indicates that it is unlikely that the LINAC can achieve its high availability requirements of $98 \%$ without astute use of equipment configuration to allow faulttolerant operation. 


\section{INTRODUCTION}

The present Spallation Neutron Source (SNS) linear accelerator (LINAC) has normal conducting (NC) modules for its entire length. Its design configuration is such that it must shut down whenever a cavity is unable to accelerate particles because of cavity or associated component failure. That is, any cavity failure will cause the LINAC to shut down. In this baseline design, a klystron and its associated equipment services eight cavities, causing the shut down of a long length of LINAC beam line whenever a klystron fails. On the other hand, there is a klystron for each cavity in one of the proposed superconducting (SC) LINAC designs. Each SC cavity has substantial reserve capability. This enables an SC LINAC to operate with multiple cavity failures, giving it a higher availability than an NC LINAC. In this study, it is assumed that an SC LINAC can continue operation as long as no two, adjacent cavity fails.

The purpose of this work is to perform a brief, rough estimate of the increase in availability in going from an NC to an SC LINAC. Equipment common to both NC and SC designs was not considered. This study limited itself to about ten generic types of equipment that are listed in Tables 1 and 2 . It is not the objective of this work to estimate overall LINAC availability, merely to estimate the difference in availability between NC and SC concepts. This work used the input data developed for the Accelerator Production of Tritium (APT) Project (ref. 1) to represent SNS design. These failure rate and repair time input data are listed in Tables 1 and 2. The LINAC design consists of a conventional, NC section at energy below - $200 \mathrm{MeV}$, and an SC section between 200 and 1,000 MeV. 
Table 1. Components and data for NC LINAC section (- 200 to $1,000 \mathrm{MeV}$ )

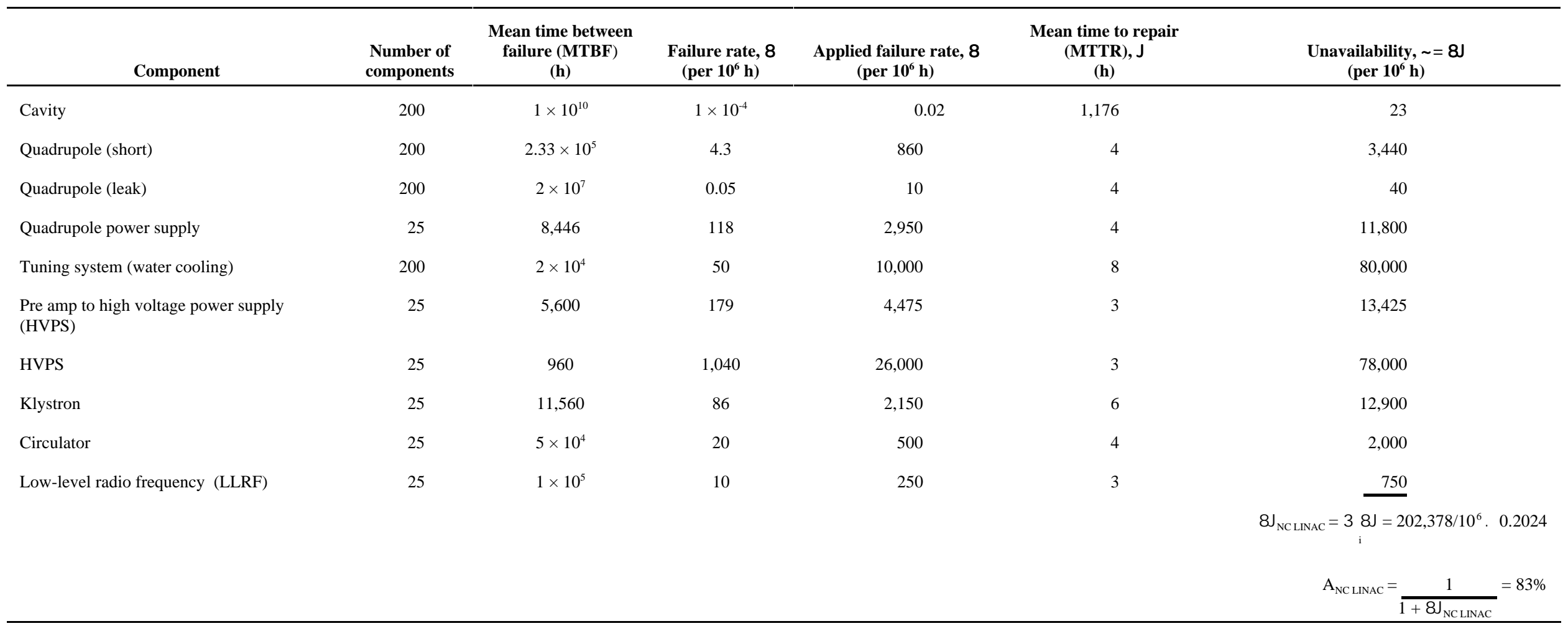


Table 2. Components and data for SC modules in LINAC section (- 200 to 1,000 MW)

\begin{tabular}{|c|c|c|c|c|c|}
\hline Component & $\begin{array}{c}\text { Number of failures required } \\
\text { to shut down LINAC }\end{array}$ & $\underset{\text { (h) }}{\text { MTBF }}$ & $\begin{array}{c}\text { Failure rate, } 8 \\
\left(1 / 10^{6} \mathrm{~h}\right)\end{array}$ & $\begin{array}{l}\text { MTTR, J } \\
\text { (h) }\end{array}$ & $\begin{array}{l}\text { Unavailability, } \\
\left(1 / 10^{6} \mathrm{~h}\right)\end{array}$ \\
\hline Cavity & 3 & $1 \times 10^{10}$ & $10^{-4}$ & 24 & 0.0024 \\
\hline Tuning system (mechanical) & 2 & $1 \times 10^{6}$ & 1 & 24 & 24 \\
\hline Vacuum valve & 2 & $1 \times 10^{8}$ & 0.01 & 24 & 0.24 \\
\hline Quadrupole & 1 & $2.33 \times 10^{5}$ & 4.3 & 4 & 17.2 \\
\hline Quadrupole power supply & 1 & 8,446 & 118 & 4 & 472 \\
\hline Vacuum pump & 2 & $2 \times 10^{4}$ & 50 & 4 & 200 \\
\hline Pre amp to HVPS & 3 & 5,600 & 179 & 3 & 536 \\
\hline HVPS & 3 & 960 & 1,040 & 3 & 3,120 \\
\hline Klystron & 3 & 11,560 & 86 & 6 & 516 \\
\hline Circulator & 3 & $5 \times 10^{10}$ & 20 & 4 & 80 \\
\hline LLRF & 3 & $1 \times 10^{5}$ & 10 & 3 & 30 \\
\hline \multirow[t]{3}{*}{ Input coupler window } & 2 & $8.2 \times 10^{4}$ & 12 & 24 & 288 \\
\hline & & \multicolumn{4}{|r|}{$\tau_{\text {sc quads }}=489 / 10^{6}=0.0004891$} \\
\hline & & & $\mathrm{A}_{\mathrm{SC} \text { LINAC }}$ & (58) sC quads & $1-58\left(489.1 / 10^{6}\right) \cdot 97.16 \% *$ \\
\hline
\end{tabular}

*There are 58 quadrupole magnets and associated power supplies in the SC section of the LINAC. Since all equipment is redundant, their contributions to LINAC downtime is assumed to be neglible. 


\section{LINAC DESCRIPTION}

\subsection{NC LINAC DESCRIPTION}

Figure 1 gives an overview of the major sections of a LINAC. That region below $200 \mathrm{MeV}$ was not modeled in this study. Because of the limited time allotted for this study, simplifying assumptions were made in modeling the LINAC. For the NC LINAC, the region between 200 and 1,000 MeV was assumed to consist of 25 identical modules, each with 8 cavities. There are ten resonant cells in each cavity. Each module contained a klystron, an associated power supply, and other associated equipment, such as a circulator and LLRF control. Each cavity contains a quadrapole magnet. Each quadrapole power supply will be manifolded to - 10 to 20 quadrapole magnets in a configuration yet to be determined. A schematic of an NC module is shown in Fig. 2. A failure of any of these components in an NC LINAC will cause the LINAC to shut down. The failure of vacuum pumps was not considered because these have multiple redundancy and are not large contributors to system failure and shutdown. Input coupler windows were not considered, although their failure could be a problem.

\subsection{SC LINAC DESCRIPTION}

It is proposed that the SNS LINAC be both NC and SC—with the transition occurring at $197 \mathrm{MeV}$. In the proposed SC portion of the SNS LINAC (that region above - $200 \mathrm{MeV}$ ), there will be 9 threecavity cryomodules in the medium beta section and 20 four-cavity cryomodules in the high beta section. A three- cavity cryomodule is shown in Fig. 3. There is a klystron, a HVPS, and associated equipment

for each cavity. Quadrapole magnets are on either end of the module, and there is a power supply associated with each quadrapole magnet. There is a vacuum pump manifold in each warm section, but as in modeling the NC LINAC, vacuum pump failures are not considered in this study. Likewise, the failure of input coupler windows are not considered. There are six resonant cells in each SNS cavity. The SC portion of the SNS LINAC cannot tolerate a whole cryomodule loss (i.e., loss of three cavities) because of very stringent beam loss requirements. For modeling simplicity, no distinction is made between medium- and high-beta cryomodules - all cryomodules are considered to be high-beta. 


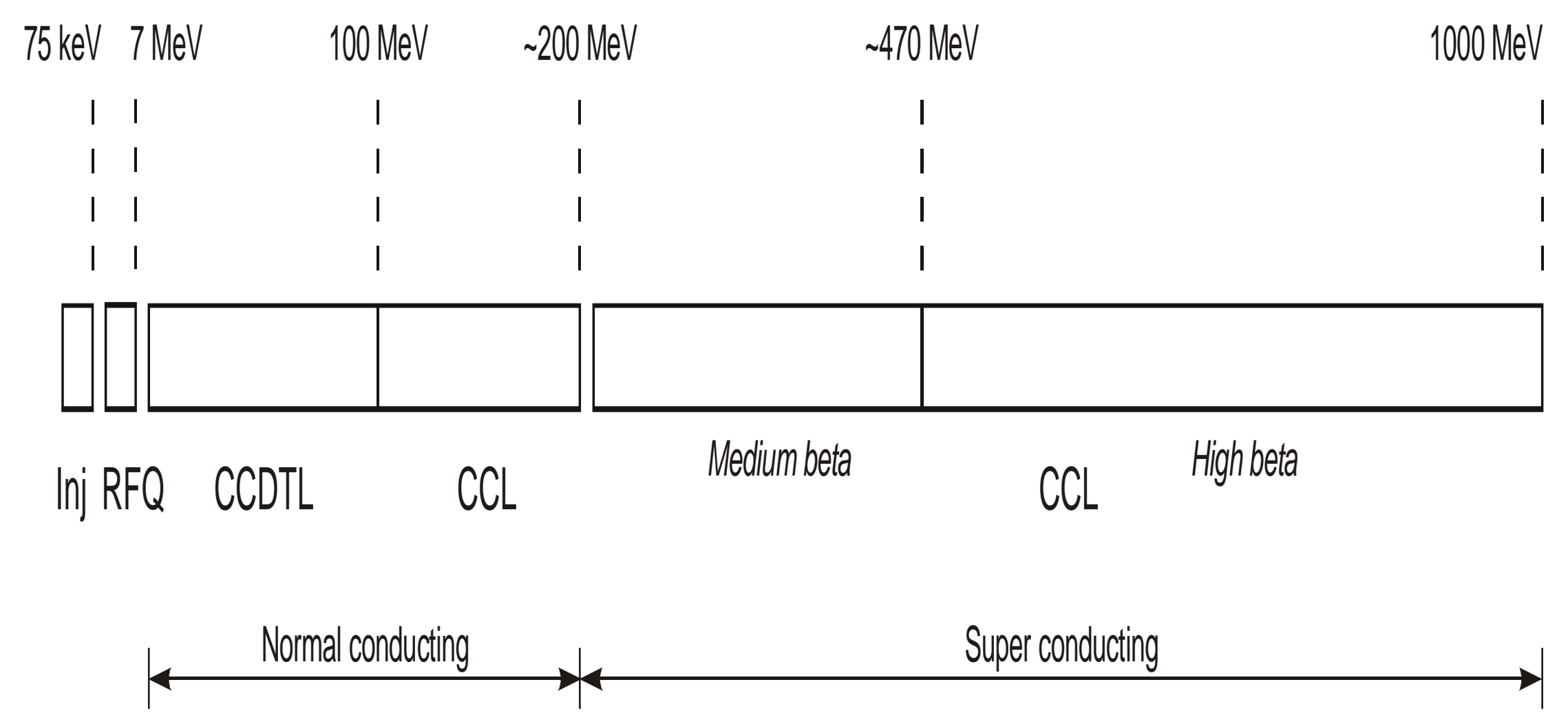

Fig. 1. Schematic of sections of SC LINAC. 


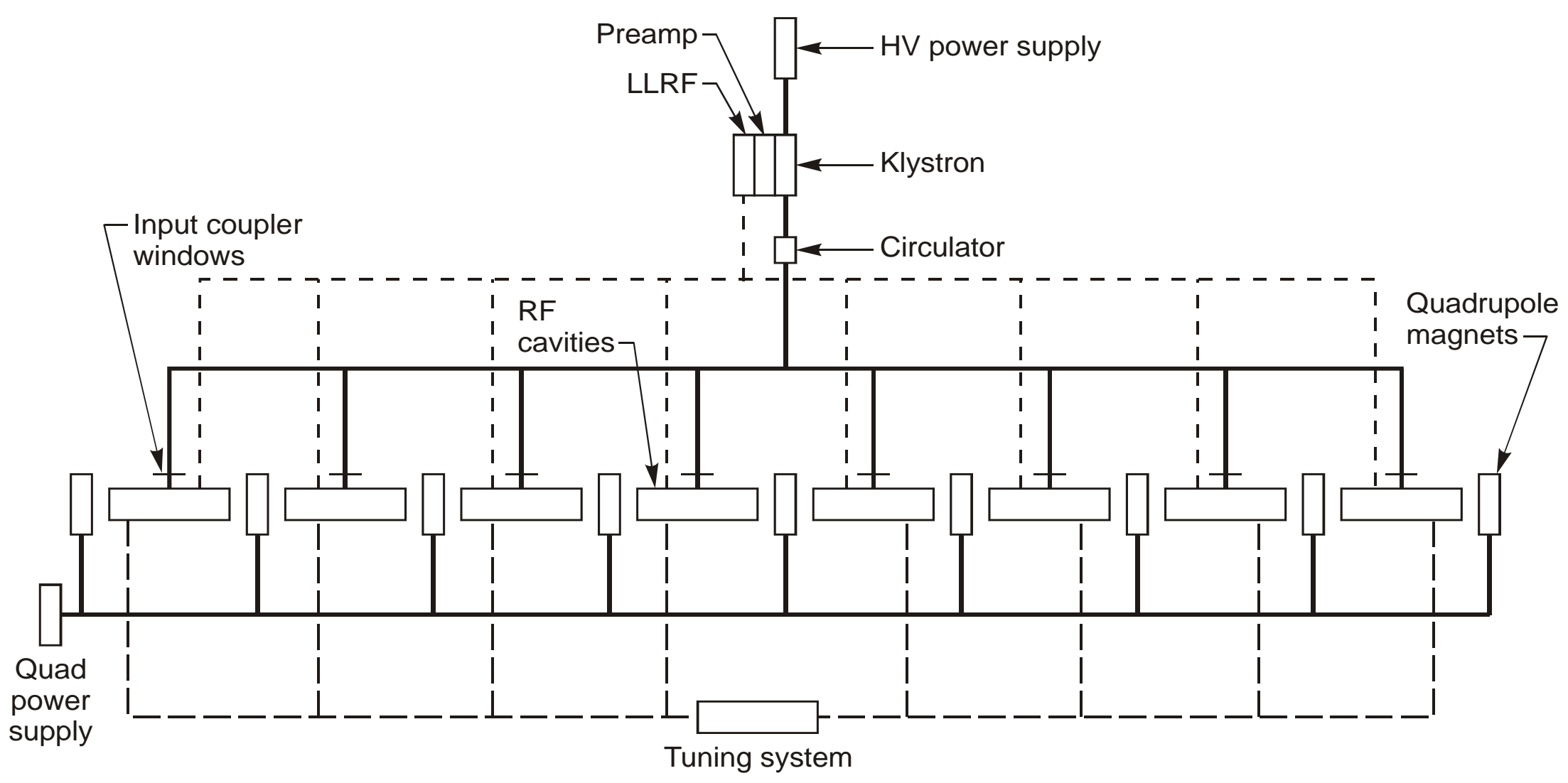

Fig. 2. Schematic of assumed NC LINAC module. (Each module contains 1 klystron servicing 8 radio frequency (rf) cavities with 10 cells per cavity, and associated equipment.) 


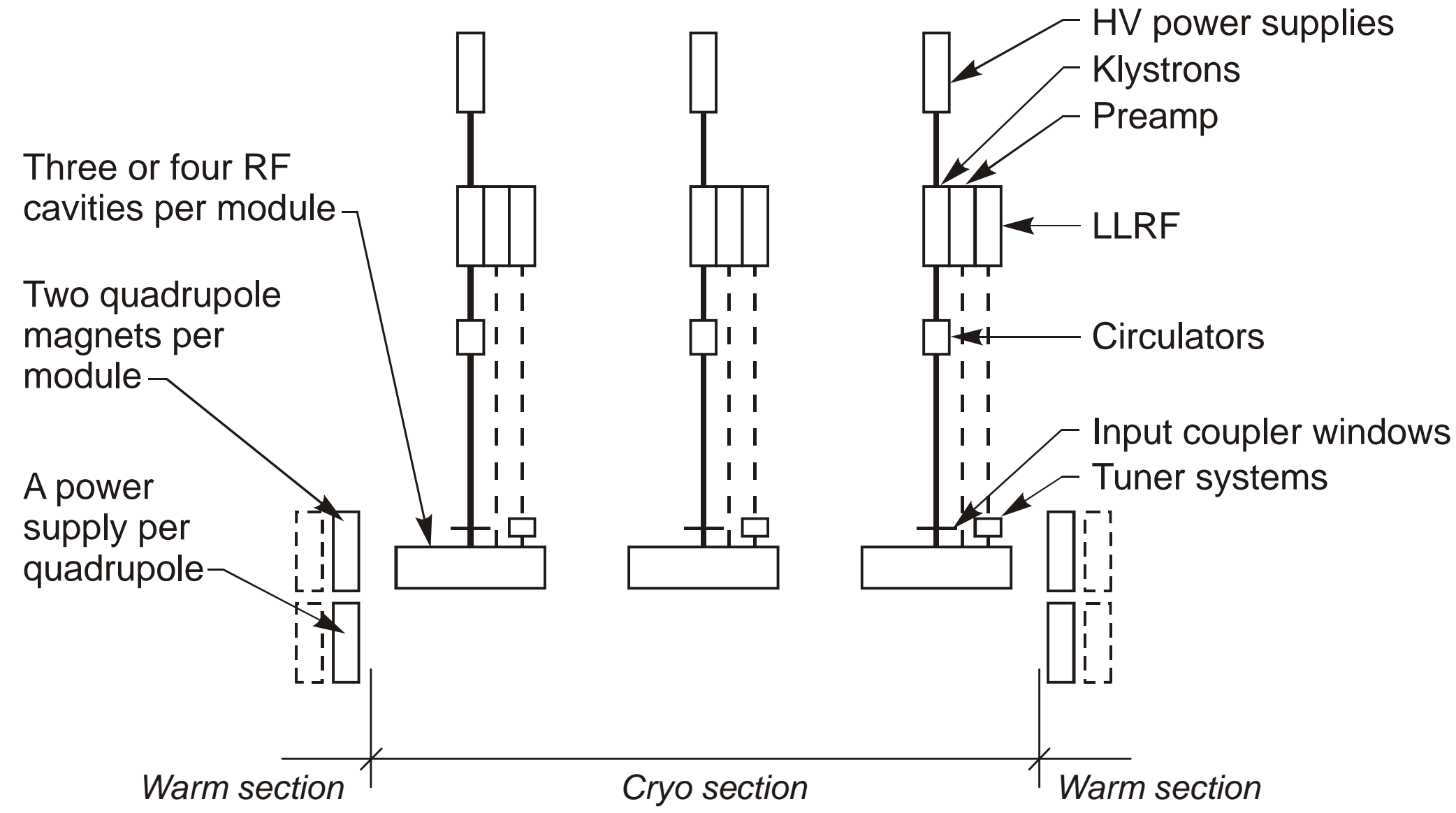

Fig. 3. Schematic of assumed SC LINAC module. (Each module contains 3 rf cavities, each driven by a klystron.) 


\section{TABULAR FAULT-TREE CALCULATIONS}

\subsection{FAULT-TREE DEVELOPMENT}

A fault tree is an analytical technique whereby an undesired system is analyzed to find credible ways in which a fault event can occur. The fault tree is a graphical model of the various parallel and sequential combinations of failures that will result in the occurrence of the predefined undesired event. A fault tree thus depicts the logical relationships of basic events that lead to the undesired event, which is the top event of the fault tree. Figure 4 is a fault tree for the LINAC arrangement where a single klystron and power supply drive each SC cavity.

A fault tree is composed of symbols that are described in detail in ref. 2. A fault tree is a complex of entities known as "gates", which serve to permit or inhibit passage of fault logic up the tree. There are two basic types of fault-tree gates: the OR gate and the AND gate. The OR gate (the symbol with the curved bottom in Fig. 4) is used to show that the output event occurs only if one or more of the input events occur. The AND gate (the symbol with the straight line at the bottom in Fig. 4) is used to show that the output occurs only if all the input faults occur. The circle describes a basic initiating fault that requires no further development. A rectangle box is an intermediate or final event that occurs because of one or more preceding event.

The fault tree is thus developed until all components of the system are identified in their basic fault states. A fault tree is not itself a quantitative model. It is a qualitative model that can be evaluated quantitatively. The result is a binary model of the systems that can be reduced to its simplest Boolean algebra form. Boolean algebra is especially important in situations involving a dichotomy: valves are either open or closed, power supplies either operate or they do not, events either occur or they do not. The fault tree is a pictorial representation of Boolean relationships among faults events that cause the top event to occur. A fault tree can always be translated into an entirely equivalent set of Boolean equations.

The fault tree is thus developed until all components of the systems are identified in their basic fault states. Equipment failure probabilities and appropriate time intervals can be assigned to determine probability values for components, subsystems, and an entire system. The quantification process involves naming of events and the transferring of all the information contained in the fault tree to event tables and coding sheets for ease in assigning data to events. 


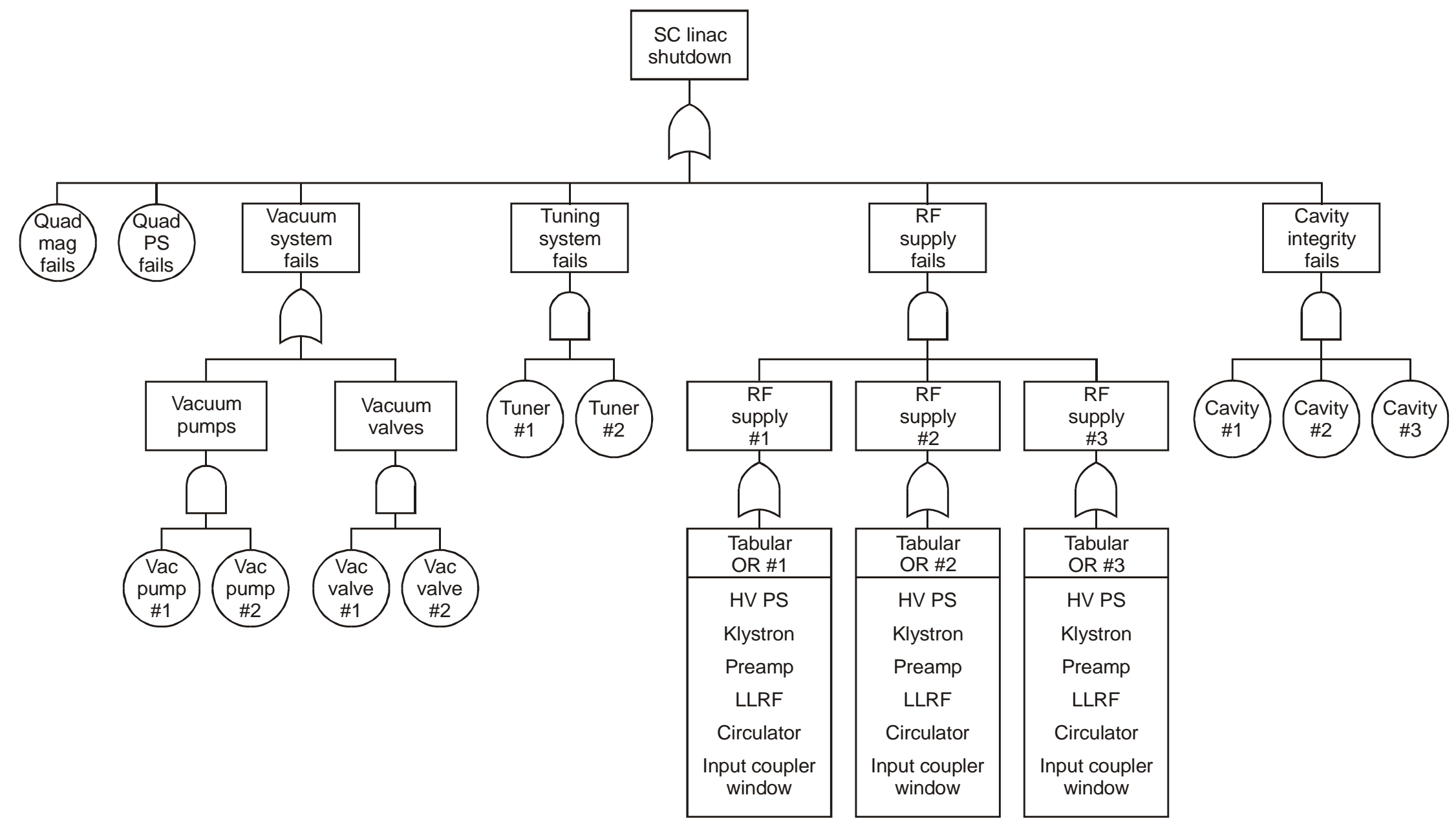

Fig. 4. Fault tree for LINAC arrangement, where every klystron drives an SC cavity. 
Since all the basic event statements on the conventional fault tree are subsequently transferred to tables, one way to reduce the analysis effort is not to put those statements on the fault tree in the first place. The first step in the abbreviated method is to enter all basic fault statements directly into fault summary tables. The second step is listing the event names in a table rather than event names within symbols. Typically, the systems that are evaluated contain a large number of events that are logically in series. Those that are not in series (e.g., AND gates) are reduced until they are a subsystem in a series. This procedure generates a tabular fault tree (ref. 3). Table 1 is an example of a tabular fault tree.

The tabular fault tree has several advantages over a conventional approach, all of which ultimately reduce the time and effort required to evaluate the system. The tabular fault tree can be readily restructured to add new subsystems and equipment. Events can be expeditiously added or crossed off, and blocks or events can be added or removed if logic changes. A typical fault tree, fully developed in the traditional way, requires much analysis, and it may require many sheets of paper to show all component fault statements. The same component faults can usually be shown on a single page or two of a tabular fault-tree table. A disadvantage of this approach is that its quickly developed logic may overlook a significant fault or that the top event may be inaccurately portrayed or calculated.

\subsection{BASIC EQUATIONS}

Tabular fault-tree calculations were performed as a "sanity check" of event-driven simulation (EDS) computer calculations. Analysis of the NC LINAC indicates that failures of any single item is cause to shut this system down. This characteristic "OR" gate represents a summation in boolean-algebra-faulttree analysis. There are a number of "AND" logic gates that require two or more events to cause a higher tier fault in SC LINAC modules. Redundant equipment represented by AND gates are collapsed, by multiplication of individual availabilities, to an equivalent equipment subsystem, which is represented by a single failure and repair time. These are then summed. These characteristics of a tabular fault tree greatly simplify the quantification of the fault tree by making the unavailability probabilities simply the sum, or the sum of products, of the probabilities of the individual failure events.

Availability is defined as the MTBF divided by the MTBF plus MTTR. Thus, 


$$
\begin{gathered}
A^{\prime} \frac{M T B F}{M T B F \% M T T R} \\
1 \frac{1}{1 \% \frac{M T T R}{M T B F}} \\
1 \frac{1}{1 \% 8 \mathrm{~J}}
\end{gathered}
$$

where 8 is the failure rate and $J$ is the MTTR for the event. The MTBF variable is the inverse of the failure rate. An exponential failure density distribution is assumed: All failures are random.

Availability is defined as one minus the unavailability variable

$$
\left.A_{\text {subsys }} \text { ' } 1 \text { ! }\right\}_{\text {subsys }}
$$

The unavailability $(\dashv$ of a single repairable component $(i)$ is approximated by

$$
\sim_{i} \cdot \text { 8j }_{i} \quad, \quad \text { if } 8 \mathrm{~J} \ll 1
$$

High system availability and short repair times are assumed in Eq. (3).

The availability of components in parallel (AND logic gates in fault-tree analysis) is represented by the product,

$$
a_{\text {subsys }}{ }^{\prime} \mathrm{A}_{i} a_{i}
$$

The unavailability of components in series is

$$
\widetilde{\sim}_{\text {subsys }}{ }^{\mathrm{I}} \mathrm{j}_{i} \widetilde{\tau}_{i}
$$

The unavailability (\}$)$ of the system is

$$
\}_{\text {sys }}{ }^{\mathrm{I}} \mathrm{j}_{i} \sim \text { subsys }
$$


Equations (4) and (5) concern only series (nonredundant) and parallel systems (fully redundant) components. In many systems these two extremes are not always applicable because there are some parts of the systems that are partially redundant. In these systems, the principle of network reduction applies: Parallel and series components are successively reduced to equivalent single components. The best way to identify parallel and series components and systems is to draw a reliability block diagram (ref. 4), perform network reduction of this diagram, and then generate a tabular fault-tree table. Eliminating the reliability diagram and going directly to the table are prone to error.

\subsection{TABULAR FAULT-TREE CALCULATIONS}

Tables 1, 2, and 3 show tabular fault-tree calculations for this evaluation. In Table 1, the specific item of equipment is listed in the first column. The failure rate for each equipment item is given in the fourth. The number of the particular part in the subsystem is listed in the second column. The application failure rate, the 5th column, is obtained by multiplying the second and fourth columns together. The MTTR for a particular part is shown. The unavailability caused by the failure of a specific part, the last column, is obtained using Eq. (3). The total unavailability is calculated using Eqs. (4), (5), and (6). The availability of the NC section of the LINAC, or for an SC module, is calculated using Eq. (1).

No attempt is made to divide the LINAC into modules for the calculation of the NC LINAC availability shown in Table 1. The calculation is based on the number of each component between 200 and 1,000 MeV. The number of parts for each component is multiplied by the failure rate, giving the applied failure rate, and this rate then is multiplied by the MTTR to give the total unavailability for that component in the system. The unavailability of all the components are summed to give the system unavailability and from that the availability.

There are 29 modules (i.e., 58 quadrupole magnets and power supplies) in the SC LINAC. The second column of Table 2 indicates that all components are either double or triple redundant, except for the quadrupole magnets and associated quadrupole power supply. A failure of a quadrupole magnet or quadrupole power supply will shut down the LINAC. Other equipment redundancy contributes a negligible amount to the total unavailability. Thus, the unavailability of an SC module is essentially the unavailability of the quadrupole magnets and associated power supplies. The unavailability of the SC LINAC system is, therefore, estimated as 58 times the unavailability of SC quadrupole magnets and power supplies. 
One proposed SC LINAC design configuration has one klystron and associated equipment servicing six cavities. This is similar to that of the NC LINAC in terms of reliability because a single klystron failure, or associated equipment, will shut down six cavities and, hence, the LINAC. The Table 1 spreadsheet is modified to calculate the availability for this SC equipment configuration, as shown in Table 3. A single failure of a quadrupole magnet or quadrupole power supply continues to shut this SC system down. A mechanical tuning device must be used for the SC LINAC instead of the water-cooling method of tuning. The number of klystrons and associated equipment must increase because a single klystron services six cavities in this SC design, whereas a klystron usually services eight cavities in the NC design. Thus, the number of these equipment increase by $8 / 6$ or from 25 to 33 klystrons. This, in turn, increases the failure rate for this system because of their greater numbers. However, the increased reliability and maintainability of the mechanical tuning method more than offset this increase. The availability for this SC equipment configuration is $86.3 \%$, only $3.3 \%$ greater than that of an NC LINAC.

The fault-tree methodology can not model the spatial constraints contained in this SC study (i.e., adjacent cavity failure in an SC LINAC will cause LINAC shutdown). Normally, at least three cavity failures are required for SC LINAC shutdown. Also, fault-tree calculations such as done here can not model time dependence or degraded equipment operation (i.e., equipment operating at $<100 \%$ design). 
Table 3. Components and data for SC LINAC with six cavities per klystron

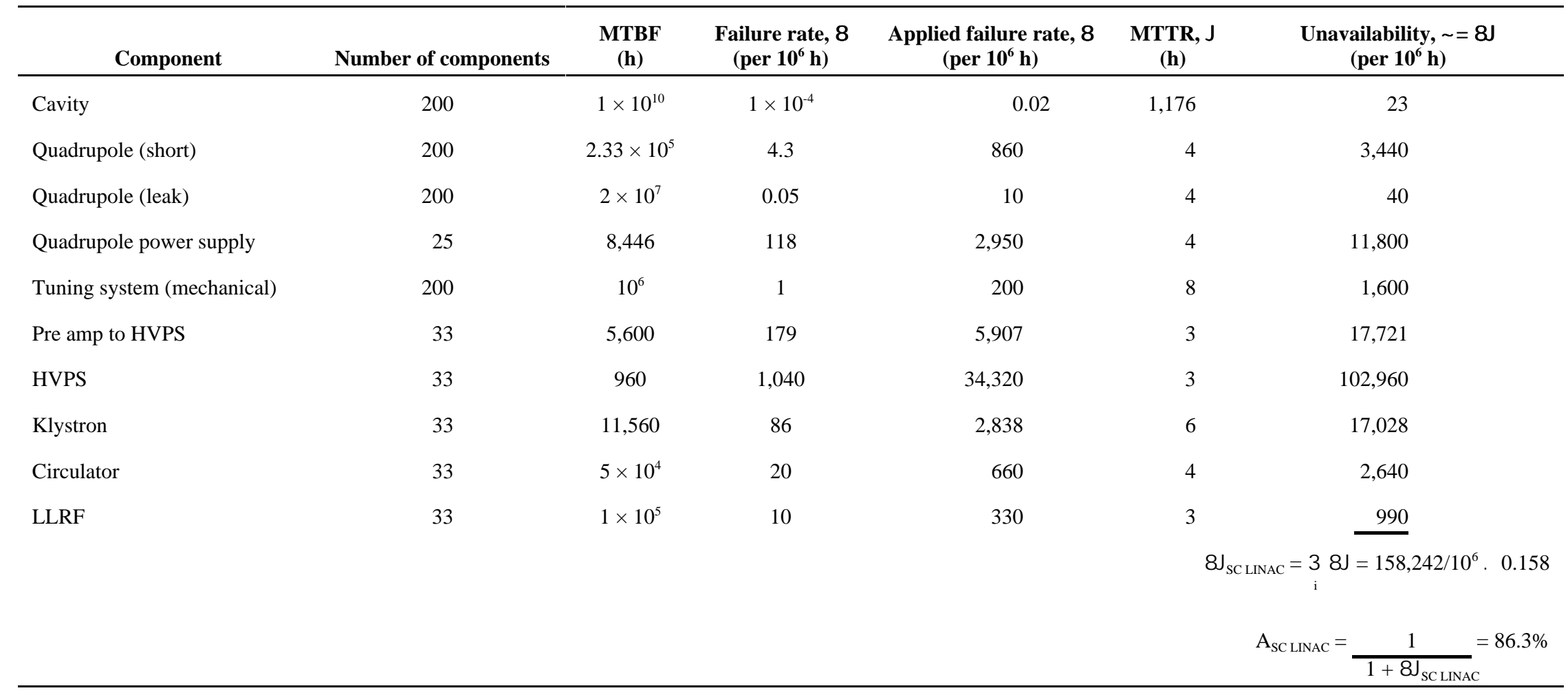




\section{COMPUTER SIMULATION OF LINAC AVAILABILITY}

\subsection{EDS COMPUTER CODE}

A computer model is needed for this problem because simple, single-point failures plus the sum of product of single-point failures on a spreadsheet cannot accurately represent the complex network configuration of equipment systems. An EDS was produced to compute SC LINAC availability from the MTBF and MTTR for subsystems composing the NC and SC modules. The EDS is a powerful tool, which carries out computations in the time domain which is able to handle complex failure logic (e.g., requirements for nonadjacent cavity failures).

Figures 2 and 3 show a schematic of the systems modeled. Any component shown in Fig. 2 or Table 1 that fails will cause the shutdown of the NC LINAC. In Fig. 2, failure of a klystron, klystron power supply, klystron preamp, circulator, or LLRF control could cause failure of a cavity. But three cavities must fail to cause the shutdown of the SC LINAC. An SC LINAC cannot operate with failure of a quadrupole or quadrupole power supply failure since three cavities are affected.

The EDS was implemented using Matlab language and computing environment in two $\mathrm{m}$-files. The EDS engine is found in supLINAC2.m, and the front-end driver of the EDS is found in a file named demosim2.m. A similar file exists to compute NC availability. The LINAC availability computer model begins by generating initial failure times for each component by sampling from an exponential distribution and sorting the failure times into an event queue. Simulation time progresses by popping each event off the top of the event queue in order and then checking to see whether shutdown criteria have been satisfied. The simulation code keeps track of which subsystems have failed. Only operational time is counted in the simulation. When the system shuts down for unscheduled repairs and scheduled maintenance, new failure times are generated, and the event queue is resorted. The cumulative time to repair each failure resulting in shutdown is saved, and availability can then be estimated by dividing the total operating time by the sum of the total operating time plus unscheduled repair time.

The simulation code allows the user to specify values of several parameters, including the total number of allowable module failures, total number of allowable cavity failures, the number of modules, minimum distance allowed between cavity failures, total operational time, number of runs, and length of the operational cycle. It is also possible to change the number of cavities per module and component MTBFs, and MTTRs.

EDS computations give an SC LINAC availability for a number of equipment configuration conditions in Fig. 5. The same simulation code gave an NC LINAC availability of $83.1 \%$. Figure 4 shows the effects of spatial failure limits and different configurations for HVPSs. Each EDS availability estimate in Fig. 5 is an average of three runs simulating 200,000 h of operation time. 
ORNL DWG 2000-73

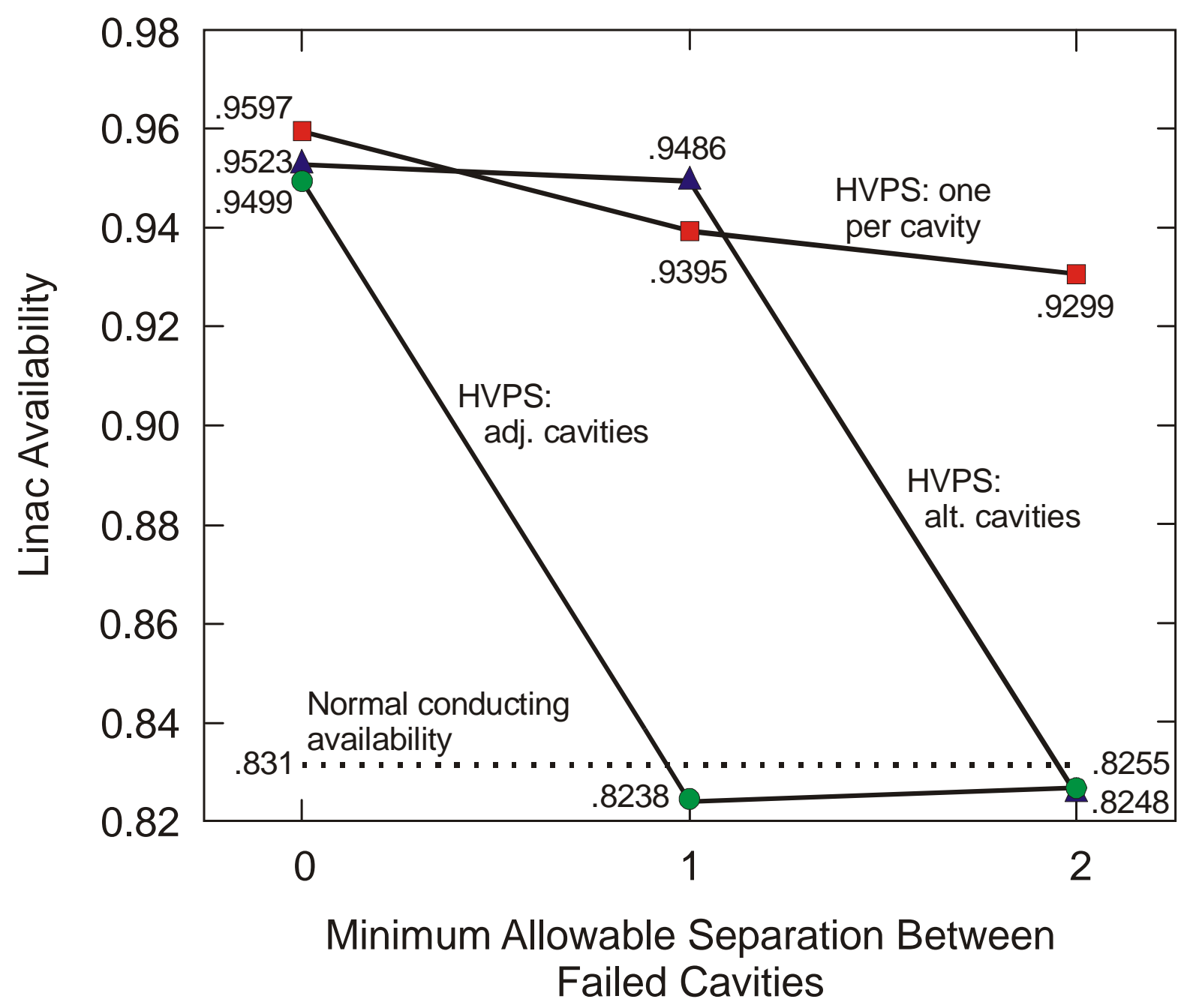

Fig. 5. The effect of klystron HVPS configuration on SC availability. 


\subsection{THE EFFECTS OF CAVITY FAILURE LOCATION AND HVPS CONFIGURATION}

A separate simulation study was made of the impact on availability of the total number of individual cavity failures allowed. For example, the APT project allows as many as seven cryomodule failures (containing two or three cavities) so long as no two are adjacent. SNS currently proposes allowing a total of only two cavity failures as long as the two cavity failures are not adjacent.

The SC LINAC is permitted to operate in degraded modes under a spatial failure criteria that allows isolated failed cavities along the LINAC beam line. The proximity rule limits the spatial distribution of failed cavities connected in series. Beam dynamics prefer failed modules to be isolated from each other instead of clustering close together. Greater separations provide more stability of the configuration. The exact limiting separation can be investigated as a free parameter in the LINAC availability model.

Conventional steady-state reliability and maintainability analysis techniques cannot be used to calculate LINAC availability spatial separation criteria. It becomes necessary to carry out calculations in the time domain. A simple EDS was written to estimate the MTBF of the SC section of a LINAC under the global failure criteria spatial separation requirements. The assumptions of the simulation model are given below.

The SC model provide 29 cryomodules connected in series. Each cryomodule has an identical configuration, failure rate, and MTTR. The operating and outage schedule are constants and do not vary according to operating conditions. Failures and unscheduled outages originating in other subsystems are not considered in the analysis. It is assumed that cavity maintenance does not occur during operating cycles and that all cavity repairs are completed during the next scheduled outage. Finally, it is assumed that all cavities are restored to working condition following an unscheduled outage due to failures in the LINAC.

The MTBF reflects the estimate for a single klystron. It is assumed that a single klystron failure shuts down a cavity. Other modeled components that shut down a cavity include circulators, transmitters, LLRFs, and tuner assemblies. The HVSP shuts down a cavity pair, while the quadrupoles and quadrupole power supplies shut down an entire cryomodule.

Figure 5 shows three curves; each contains three data points that represent different levels of separation between failed cavities. The numerical value is the minimum separation between failed cavities that allow the LINAC to continue to operate. For example, a minimum separation of zero is the most nonrestrictive condition because adjacent failures are permitted. If the separation value is one, then at least a single working cavity must separate two failed cavities. 
Each curve in Fig. 5 represents a different HVPS configuration. The curve with circular data points represent a configuration where a single HVPS serves a pair of adjacent cavities. The curve with triangular data points shows availabilities resulting from a configuration where a single HVPS serves alternating cavities. In this HVPS configuration, a single HVPS serves the first and third cavities in a cryomodule, and another HVPS serves the second and fourth cavities in the same cryomodule. The curve with squares represents a configuration where each klystron is paired with an HVPS. This configuration requires four HVPSs per four cavity cryomodule, in contrast to the two HVPS systems used in the other condition.

This simulation is a first-order approximation of the SNS SC LINAC reliability, but the results demonstrate the sensitivities of system availabilities to a type of cavity fault tolerance: cavity proximity. It can be seen from Fig. 4 that nonrestrictive proximity criteria can lead to gains in SC availability in all three configurations, compared to NC availability. The availability gain expected when spatial distribution criteria are relaxed is dramatic when the HVPS serves adjacent cavities. An HVPS serving alternating cavities is more tolerant to spatial limitations as long as the required separation does not exceed one cavity. This is easy to see in Fig. 4 because the two configurations that use two HVPS systems per cryomodule differ only in the availability achieved when a single working cavity is required between failed cryomodules. Finally, we note that when the HVPS is paired with a klystron (the curves with square data points), LINAC availability is much less sensitive to spatial distribution criteria. 


\section{DISCUSSION OF RESULTS}

The study demonstrates that there is not an inherent availability advantage of an SC LINAC over an NC LINAC. It is possible to realize substantial gains in availability with an SC LINAC, but whether the gains are realized is highly dependent upon the equipment configuration. The number of klystrons and associated equipment servicing an rf cavity or cavities should not be greater than the allowed number of cavity failures to continue operation. The EDS shows that the HVPS in particular is highly sensitive to the availability of the SC LINAC, especially when an HVPS services two or more klystrons. Most of the simulation conditions shown in Fig. 5 produce availability estimates in the 0.95 to 0.96 range, which is a substantial increase over the NC availability of 0.83. The EDS estimate was produced from a single simulation run representing $1,000,000 \mathrm{~h}$ of operation time. The estimated availability gain for the SC option is therefore from 3-13\%. This is as expected since any failure would shut down an NC. However, when the HVPS serves adjacent cavities, but nonadjacent cavity failures are required to continue LINAC operation, estimated availability plummets to just below the calculated NC availability.

Tabular fault-tree calculations and computer simulation modeling in the NC case give the same answer to within one-tenth of a percent. It is seen that results of the NC LINAC are virtually identical. Spreadsheet calculations shown in Table 2 give an SC availability of 97\%. These tabular fault-tree calculations correspond closely to the EDS condition allowing zero separation between modules and one HVPS per cavity. That estimate was $96 \%$ so that tabular fault tree and EDS calculations disagree by only $1 \%$. However, the agreement may be somewhat coincidental, since there are significant differences between the assumptions used for tabular fault-tree calculations and EDS. Some of these differences are noted below:

1. SPATIAL EFFECTS. EDS can model separation distances between failures (e.g., it can prohibit adjacent failures).

2. OPERATION SCENARIOS (Time). EDS can account for tasks during scheduled downtime. For example in this exercise, EDS assumes repair of all items that failed while operating are repaired during scheduled shutdown to good-as-new condition.

3. EQUIPMENT CONFIGURATION. In EDS modeling for example, an individual HVPS can service a single klystron, adjacent klystrons, every third klystron, etc. Such cannot be done with tabular fault-tree calculations.

4. FAILURE COMBINATIONS. Tabular fault-tree calculations done without first developing reliability block diagrams, conventional fault trees, etc. do not account for failures of different items together, causing shutdown. For example, tabular fault-tree calculations in this exercise identified three klystrons as having to fail to cause LINAC shutdown because three klystrons drive three cavities. However, failure of a klystron, a preamp, and an LLRF each in a different cavity line will also cause LINAC shutdown and this is not modeled in these tabular fault-tree calculations. Thus, these tabular fault-tree calculations estimate an availability that is too high. 
One proposed SC LINAC design configuration has one power supply driving six cavities. In this configuration, any component failure will likely cause the LINAC to shut down. The tabular fault-tree calculated estimated availability for these conditions is $86.3 \%$. This higher-than- $83 \%$ NC availability results from the higher availability of the SC tuner system.

In conclusion, tabular fault-tree calculations are generally accurate and efficient in situations during which any failure will cause system shutdown. Any system that can operate with failed components—can have redundancy, or degraded operation—requires detailed modeling, such as EDS.

This analysis did not include an evaluation of the NC and SC input coupler windows. Each type of LINAC uses different window designs, but both are redundant, and it is assumed that the reliability is about the same for both concepts. Future analysis should evaluate the availability if input coupler windows. Likewise, the vacuum system was not evaluated during this analysis.

The rf turning systems are very different in NC and SC LINACs. In the NC LINAC, the temperature of the cavity cooling water is controlled to tune the rf cavities. On the other hand, the SC LINAC uses a mechanical tuner system. The reliability of the NC LINAC tuner is much less than that of the SC tuner system: The NC tuner assembly failure rate is 50 failures $\left(1 \times 16^{6} \mathrm{~h}\right)$, as compared to an assumed failure rate of $1 /\left(1 \times 10^{6} \mathrm{~h}\right)$ for the SC modules. A significant proportion of the increase in availability for the SC LINAC results from the much lower failure rate for the tuner resonant control system in the SC LINAC. Another substantial part is due to the characteristic that multiple cavities must fail to shut down the SC LINAC.

The failure rate and repair data input to the calculations are uncertain. However, if failure rates are high, by say $30 \%$, they will be high for both NC and SC design cases, and the error from the difference in computed availabilities will largely disappear.

The major conclusions of this study are as follows:

- An SC LINAC availability is not inherently higher than that of an NC LINAC. SC availabilities can be made substantially higher than NC LINAC availabilities if the design capitalizes on advantages offered by certain equipment configurations.

- Quadrupole magnets and power supply are primary contributors to SC LINAC availability.

- Power supply to klystrons and tuner resonance control system are primary contributors to NC LINAC unavailability.

If one were to believe that the input data are even remotely accurate, since the design criteria is that the LINAC operate at $98 \%$ availability, one concludes that the SNS will not achieve its availability requirement unless clever use is made of equipment-configuration, failure-tolerate technology. 


\section{ASSUMPTIONS}

\subsection{FAULT-TREE CALCULATIONS}

1. Assume three cavities must fail to shut down SC LINAC - triple redundancy, so this equipment gives neglible contribution to unavailability.

2. That equipment where two must fail to shut down three cavities are ignored-double redundancy. For example, it is assumed that one HVPS services two klystron. Thus, two HVPS must fail to reuse at least three cavities to fail.

3. Quadrupole magnets and quadrupole power supplies dominate SC LINAC unavailability. All other equipment is ignored in calculations.

4. It is assumed that a single rf cavity failures causes an NC LINAC to shut down.

5. To be able to continue to operate with cavity failure in the SC case, a software system must be developed to sense failed cavities and to automatically rephase and adjust voltages to compensate for the lost energy gain.

6. The same failure rates and repair times are assumed for both the SC and NC concepts. This assumption is conservative in that the larger number of klystrons for the SC LINAC design will have a lower power per klystron. This should result in lower failure rates and higher availability for SC klystrons. This assumption avoids charges that the data were manipulated to draw any particular convulsion.

7. Repairs are made only during scheduled shutdowns for failures that can be tolerated while a run continues.

8. An exponential failure rate distribution is assumed. That is, the failure rate is assumed constant in time.

\subsection{EDS ASSUMPTIONS}

1. Components are not repaired during the operational cycle or unscheduled maintenance to repair systems other than the LINAC.

2. Component repairs are always completed during the next scheduled maintenance.

3. All cryomodules are functionally identical with respect to failure rates and MTTR.

4. The operation-downtime cycle is followed exactly.

5. Unscheduled repairs occur in parallel with infinite resources (i.e., simultaneous repair of all failures) so that the time to repair a failure is equal to the longest repair time of all failed components. 
6. All failed components are repaired during unscheduled downtime.

7. Unscheduled repairs that extend into scheduled downtime are always completed before the end of the scheduled maintenance period. 


\section{REFERENCES}

1. K. Kern, Accelerator Availability Calculation Report, PPO-P00-P-CLC-00048, Accelerator Production of Tritium Project.

2. U.S. Nuclear Regulatory Commission, Fault Tree Handbook, NUREG-0492, 1981.

3. U.S. Nuclear Regulatory Commission, PRA Procedures Guide, Sect. 3.6, NUREG/CR-2300, National Technical Information Service, Springfield, Virginia, September 1981.

4. R. Billinton and R. N. Allan, Reliability Evaluation of Engineering Systems, p. 73, Plenum Press, New York and London, 1983. 